\title{
2-D anisotropic magnetotelluric numerical simulation under undulating terrain based on meshfree radial point interpolation method
}

\author{
Tingzhe Huang ,Yanju Ji*, Wanyu Huang, Tonglin Li \\ College of Instrumentation and Electrical Engineering, Key Laboratory of Geo-Exploration Instrumentation, Ministry of \\ Education, Jilin University, China \\ jiyj@jlu.edu.cn, tingzhe_huang@163.com, huangwy15@mails.jlu.edu.cn, litl@jlu.edu.cn
}

\begin{abstract}
In view of the problem that widespread existing undulating terrain and anisotropic in the actual geophysical prospecting, we propose a numerical simulation method which only needs discrete nodes to construct the shape function. Assuming the electrical spindle axis of underground anisotropic medium is perpendicular to the plane, another is parallel to the plane, we construct the conductivity tensor model. From the 2-D anisotropic magnetotelluric boundary value problem, we derive the corresponding equivalent linear equation based on radial point interpolation meshfree method, the shape function is constructed by MQ-RBF function, and the coefficient matrix is compressed and stored by the MATLAB's own sparse storage function. The linear equations are solved by BICGSTAB algorithm with incomplete $\mathrm{LU}$ decomposition. The simulation results show that the undulating terrain severely affects the distribution of underground electromagnetic field and greatly changes the original anomaly characteristics, topography effect on the observation data is far greater than the influence of the anisotropy coefficient itself.
\end{abstract}

Keywords-anisotropic; magnetotelluric; undulating terrain; meshfree method;

\section{INTRODUCTION}

The interior of the earth's structure and property are the core content of geophysical research. In the 21 st century, earth science is the era of medium, structure and deep transverse inhomogeneity and anisotropy. Along with the development of modern observation technology and the improvement of cognition level, the anisotropy of media has gradually attracted people's attention, which has become a hot spot in the research of geophysics. China is a mountainous country, mountainous area accounts for about $2 / 3$ of total land area, the terrain is one of the main factors that affect the distribution of electromagnetic field. So only deeply understanding the propagation characteristics of electromagnetic waves in anisotropic media and undulating terrain can we get more close results to the actual geological interpretation and lay the foundation of geophysical inversion.

The magnetotelluric (MT) sounding is a kind of geophysical method to study the interior electrical structure of the earth by observing the natural alternating and global distributed electromagnetic field, it is widely used in the study of the earth's crust and upper mantle. Josef et al (2007) used finite difference method (FDM) to simulate the twodimensional anisotropic magnetotelluric. Li Y G et al (2008) used adaptive meshes to make an improvement on the 2-D anisotropic magnetotelluric response and achieved a good result $^{[1]}$. Based on the assumption Cagniard plane wave theory, Qin L J et al (2013) derived the magnetotelluric analytic solution of diagonal anisotropic medium ${ }^{[2]}$.

In the 20th century, the finite element method (FEM) has opened up a new era of numerical calculation, which deeply affects every branch of engineering physics. However, the finite element method, which is very mature in theoretical basis and error estimation, has been more and more challenged with the further development of the computational domain. In order to solve these problems, meshfree method based on the node topology connection to construct the approximation function arises. As a new numerical method, meshfree method is a complement and development of traditional numerical methods, which has been widely concerned in recent years. In the field of geophysics, Jia X F et al (2006) used the element free Galerkin method (EFGM) to simulate the seismic wave ${ }^{[3]} . \mathrm{J}$. Wittke and B. Tezkan (2014) simulated magnetotelluric by using meshfree local Petrov-Galerkin method (MLPG), their results show that even the nodes are randomly distributed, the meshfree method also achieved a good simulation result ${ }^{[4]}$. Li J J et al $(2014,2015)$ studied the magnetotelluric coupling algorithm of meshfree and finite element method and reviewed the research progress of meshfree method in geophysics ${ }^{[5]}$.

In this paper, we assume the electrical spindle axis of underground anisotropic medium is perpendicular to the plane, another is parallel to the plane, and then we construct the conductivity tensor model of anisotropic medium. We deduce the corresponding meshfree method equivalent linear equation based on the magnetotelluric boundary value problem. We also calculate some typical undulating terrain anisotropic models and analysis its response characteristics. The advantage of meshfree method to calculate complex models is verified, it will provide a new reference for the numerical simulation of complex terrain and complex medium.

\section{THEORY}

Fig. 1 is the schematic diagram of nodes and computing points in meshfree method. In the solution domain $\Omega$, the 
radial point interpolation method (RPIM) expression of field variable $u(\boldsymbol{X})$ at point $\boldsymbol{X}^{T}=(x, y)$ is:

$$
u(\boldsymbol{X})=\sum_{i=1}^{n} R_{i}(\boldsymbol{X}) a_{i}+\sum_{j=1}^{m} p_{j}(\boldsymbol{X}) b_{j}=\boldsymbol{R}^{\mathrm{T}}(\boldsymbol{X}) \boldsymbol{a}+\boldsymbol{P}^{\mathrm{T}}(\boldsymbol{X}) \boldsymbol{b}
$$

Where $R_{i}(X)$ is radial basis function (RBF), $n$ is the number of RBFs, $P_{j}(X)$ is the basis function of spatial coordinate, $m$ is the number of polynomial basis functions, $a_{i}$ and $b_{j}$ are undetermined constants. We use MQ function as $\mathrm{RBF}$ in this paper.

However there are $(n+m)$ variables in (1), thus $m$ more constraint conditions are required to solve the equation, so we add $m$ constraint conditions:

$$
\sum_{i=1}^{n} p_{j}\left(\mathbf{x}_{i}\right) a_{i}=\mathbf{P}^{\mathrm{T}} \mathbf{a}=\mathbf{0}, \quad j=1,2, \mathrm{~L}, m
$$

Uniting (1) and (2), we can obtain the following matrix equation:

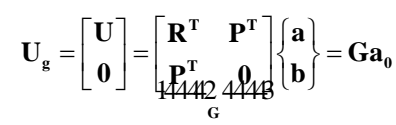

Solving (3), we can obtain:

$$
\mathbf{a}_{0}=\left\{\begin{array}{l}
\mathbf{a} \\
\mathbf{b}
\end{array}\right\}=\mathbf{G}^{-1} \mathbf{U}_{g}
$$

Equation (1) can be rewritten as:

$$
u(\mathbf{x})=\mathbf{R}^{\mathrm{T}}(\mathbf{x}) \mathbf{a}+\boldsymbol{P}^{\mathrm{T}}(\mathbf{x}) \mathbf{b}=\left\{\mathbf{R}^{\mathrm{T}}(\mathbf{x}) \boldsymbol{P}^{\mathrm{T}}(\mathbf{x})\right\}\left\{\begin{array}{l}
\mathbf{a} \\
\mathbf{b}
\end{array}\right\}=\boldsymbol{\Phi}_{g}^{\mathrm{T}}(\mathbf{x}) \mathbf{U}_{g}
$$

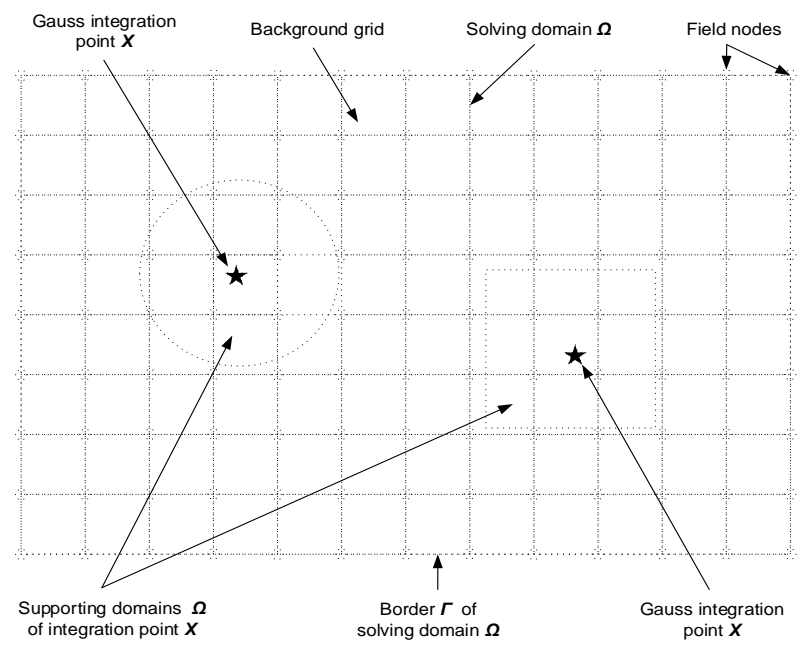

Fig. 1. Schematic diagram of nodes and computing points (Gauss points)

The RPIM shape function in (5) can be expressed as:

$$
\begin{aligned}
& \boldsymbol{\Phi}_{g}{ }^{\mathrm{T}}(\mathbf{x})=\left\{\mathbf{R}^{\mathrm{T}}(\mathbf{x}) \boldsymbol{P}^{\mathrm{T}}(\mathbf{x})\right\} \mathbf{G}^{-1} \\
& =\left\{\begin{array}{lllllll}
\phi_{1}(\mathbf{x}) & \phi_{2}(\mathbf{x}) & \mathrm{L} & \phi_{n}(\mathbf{x}) & \phi_{n+1}(\mathbf{x}) & \mathrm{L} & \phi_{n+m}(\mathbf{x})
\end{array}\right\}
\end{aligned}
$$

Assuming the conductivity of the parallel plane is $\sigma_{/ /}$, the conductivity of the vertical plane is $\sigma_{\perp}$, the strike is along $z$ axis, the vertical direction is $y$ axis, the 2-D anisotropic MT boundary value problem is summarized as follows:

$$
\left.\begin{array}{ll}
\nabla \cdot(\tau \nabla u)+\lambda u=0 & \in \Omega \\
u=1 & \in A B \\
\frac{\partial u}{\partial n}+k u=0 & \in A C, B D \\
\frac{\partial u}{\partial n}=0 & \in C D
\end{array}\right\}
$$

Where $k=\sqrt{-\mathrm{i} \omega \mu \sigma_{/ /}}, u$ is the field function to be solved, upper boundary $A B$ is essential boundary, left and right boundary $A C, B D$ are natural boundary, lower boundary $C D$ is mixed boundary. For the TE mode, the study area includes air and ground, where $u=E_{z}, \tau=\frac{1}{\mathrm{i} \omega \mu}, \lambda=\sigma_{/ /}-\mathrm{i} \omega \varepsilon, \omega$ is the circular frequency, $\mu$ is the medium permeability. For the TM mode, the study area is underground space, $u=H_{z} \lambda=i \omega \mu, \tau$ is the conductivity symmetric tensor, $\tau=\left(\begin{array}{ll}\tau_{11} & \tau_{12} \\ \tau_{21} & \tau_{22}\end{array}\right)$

$$
\left.\begin{array}{l}
\tau_{11}=\left(\frac{1}{\sigma_{/ /}-i \omega \varepsilon} \sin ^{2} \alpha+\frac{1}{\sigma_{\perp}-i \omega \varepsilon} \cos ^{2} \alpha\right) \\
\tau_{22}=\left(\frac{1}{\sigma_{/ /}-i \omega \varepsilon} \cos ^{2} \alpha+\frac{1}{\sigma_{\perp}-i \omega \varepsilon} \sin ^{2} \alpha\right) \\
\tau_{12}=\tau_{21}=-\frac{1}{2}\left(\frac{1}{\sigma_{/ /}-i \omega \varepsilon}-\frac{1}{\sigma_{\perp}-i \omega \varepsilon}\right) \sin 2 \alpha
\end{array}\right\}
$$

Where $\alpha$ is the angle between the electric principal axis of anisotropic media and the ground coordinate system.

The equivalent functional for 2-D anisotropic MT boundary value problem is:

$$
F(u)=\int_{\Omega}\left[\frac{1}{2} \tau(\nabla u)^{2}-\frac{1}{2} \lambda u^{2}\right] d \Omega+\int_{C D} \frac{1}{2} \tau k u^{2} d \Gamma
$$

Calculating the partial derivative of the functional $F(u)$, and bring $u(\mathbf{x})=\boldsymbol{\Phi}^{\mathrm{T}}(\mathbf{x}) \boldsymbol{U}$ into its derivative, let the partial derivative be 0 , so we can obtain the following matrix expression:

$$
K u=b
$$

Where

$$
\begin{gathered}
K=K_{1}-K_{2}+K_{3} \\
=\int_{\Omega} \tau\left[\left(\frac{\partial \Phi_{i}}{\partial x}\right)\left(\frac{\partial \Phi_{j}}{\partial x}\right)+\left(\frac{\partial \Phi_{i}}{\partial y}\right)\left(\frac{\partial \Phi_{j}}{\partial y}\right)\right] d \Omega-\int_{\Omega} \lambda \Phi_{i} \Phi_{j} d \Omega+\int_{C D} \tau k \Phi_{i} \Phi_{j} d \Omega \\
u=\left\{\begin{array}{llll}
u_{1} & u_{2} & \mathrm{~L} & u_{n}
\end{array}\right\}^{\mathrm{T}}, b=\left[\begin{array}{llll}
0 & 0 & \ldots & 0
\end{array}\right]
\end{gathered}
$$

Upper boundary $A B$ is essential boundary, we suppose its field value is 1 unit, in order to load the essential boundary, the diagonal element of coefficient matrix $K$ and corresponding right term $b$ are revised as $\alpha K_{i i}, \alpha$ is a larger number. This is much simpler in dealing with the essential boundary.

Linear equation (10) is a large sparse equation. In order to save memory space, we use MATLAB's sparse storage function to compress the coefficient matrix. On the other hand, in order to improve the computation speed, the BICGSTAB 
(biconjugate gradient stabilized method) algorithm is used to solve the linear equation with the incomplete LU factorization as the preconditioner. BICGSTAB method has a fast convergence rate and low computational cost, it is an effective method for solving large sparse linear equations.

\section{Model CALCULATION}

In order to verify the correctness of the algorithm, a layered model is established which is shown in Fig. 2. The lateral size of solving domain is $20 \mathrm{~km}$, and the longitudinal length is $20 \mathrm{~km}$. The transverse longitudinal nodes are uniformly distributed, the transverse spacing is $200 \mathrm{~m}$, and the longitudinal spacing is $100 \mathrm{~m}$.

Fig. 3 is the apparent resistivity and phase comparison between meshfree solution and analytical solution for layered model at the ground center point. It can be seen from the figure that the numerical solution and the analytical solution at each frequency are highly consistent, and the root mean square (RMS) error is no more than $1 \%$. It fully validates the effectiveness of meshfree method to solve magnetotelluric problem and lays a foundation for the undulating terrain and anisotropic magnetotelluric forward modeling.

We respectively establish two anisotropic circular abnormal body models in the homogeneous medium under the undulating terrain, as shown in Fig. 4. Using the same meshfree parameters with Fig. 3.The background resistivity of homogeneous space are respectively $10 \Omega \cdot m, 100 \Omega \cdot m$, the parallel resistivity of the circular abnormal body are respectively $100 \Omega \cdot m, 10 \Omega \cdot m$. Abnormal body center is located in $(0,4000 \mathrm{~m})$, its radius is $1000 \mathrm{~m}$. The simulation results under different anisotropic coefficients can be obtained by changing the vertical direction resistivity.

Fig. 5 is the simulation results of apparent resistivity for different anisotropic coefficients at the same frequency. Due to TE polarization mode is not sensitive to anisotropic geoelectric structure, in this paper, we mainly study the TM polarization mode anisotropic response characteristics.

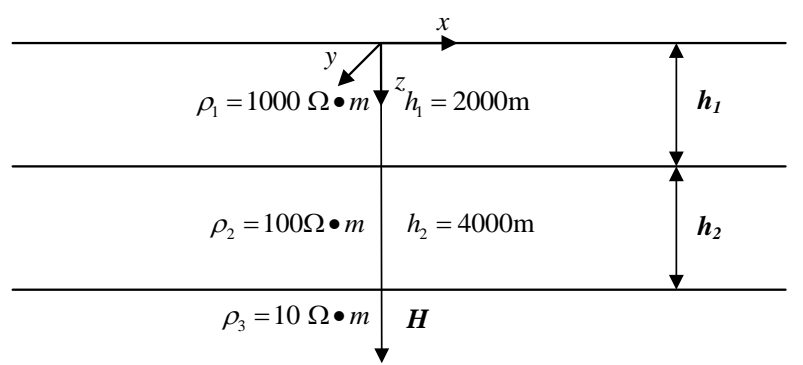

Fig. 2. Layered model schematic diagram
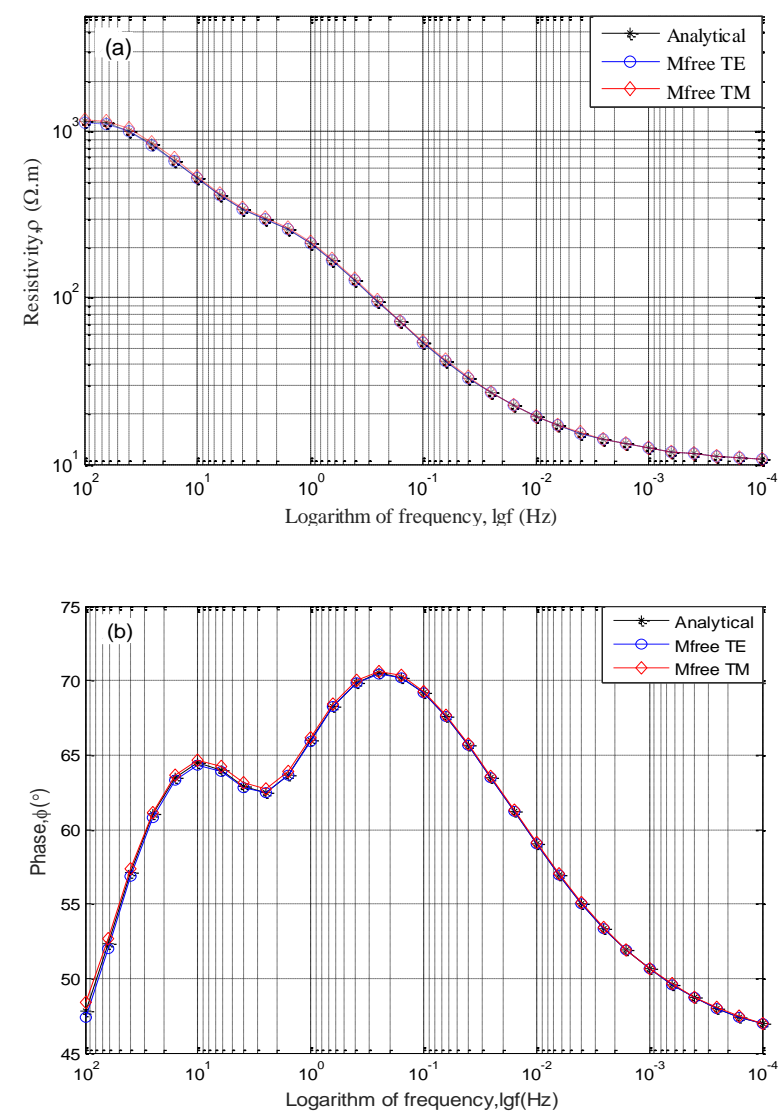

Fig. 3. Comparison between meshfree solution and analytical solution for the layered model (a) Apparent resistivity (b)Phase

It can be seen from the figure that there is a mirror image relationship between the topography and the TM mode apparent resistivity curve, at the inflection point of the terrain, the apparent resistivity changes dramatically, so when interpretating the magnetotelluric data under undulating terrain, we should put a high degree of attention to the impact of the terrain. Horst terrain makes the magnetotelluric apparent resistivity show low resistivity anomaly. When containing a high resistance anomaly body, the influence of topography on the TM polarization mode is much larger than the anisotropy itself. When changing the anisotropy coefficient of the abnormal body, the terrain also almost covers the influence of anisotropy coefficient. So it is not conducive to observe abnormal body in the horst terrain.
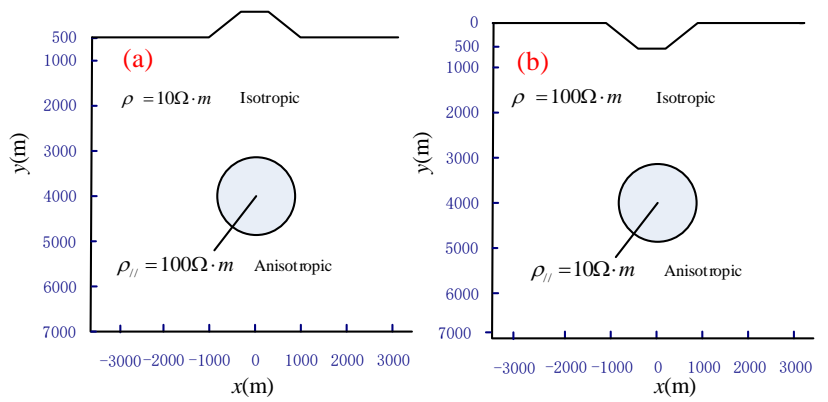

Fig. 4. Anisotropic abnormal model under undulating terrain 

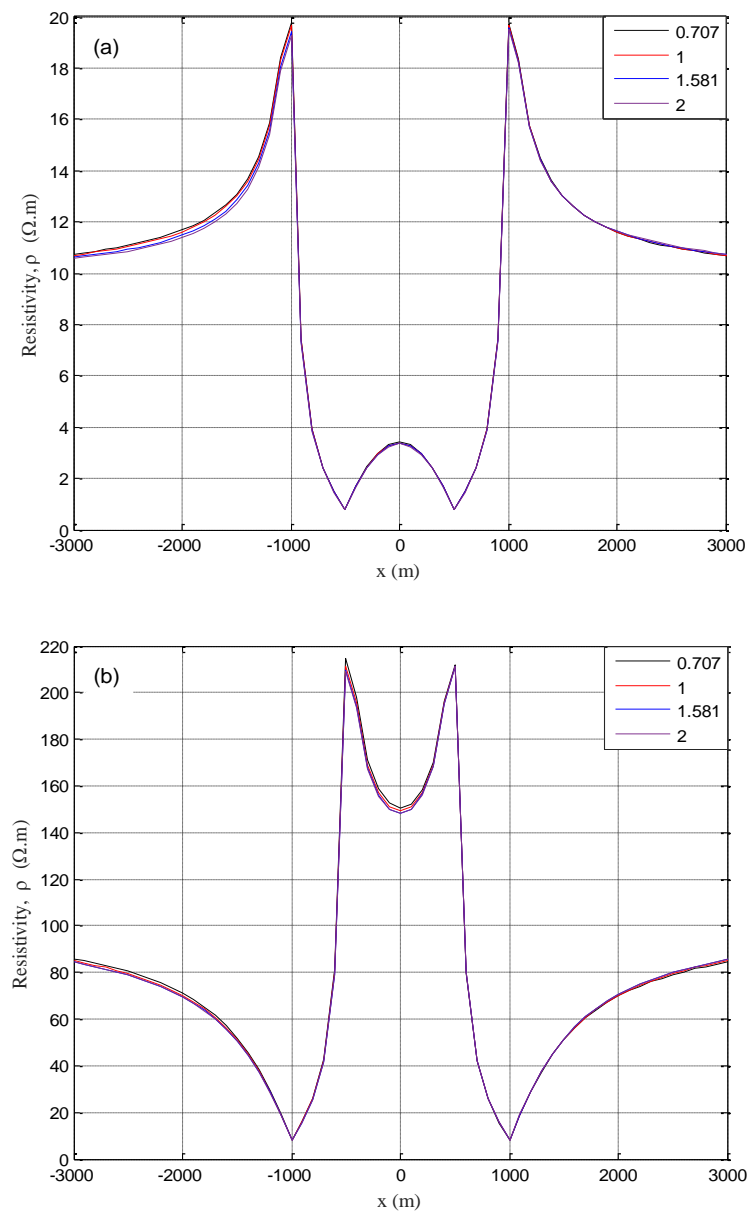

Fig. 5. Apparent resistivity of different anisotropic coefficients (a) Horst terrain with high resistivity anomaly (b) Graben terrain with low resistivity anomaly

Graben terrain makes the magnetotelluric apparent resistivity show high resistivity anomaly. The graben topography itself covers up the abnormal information for high resistivity abnormal body, but for low resistivity abnormal body, the TM polarization mode shows some certain resolution. So when dealing with MT sounding, we should try to choose the depressed area and avoid the salient region. When analysing the MT sounding data under undulating terrain with anomaly, special attention should be paid to the impact of terrain.

\section{CONCLUSIONS}

- The meshfree method based on radial basis function is out the limitation of mesh, the shape function is smooth, essential boundary condition is very easy to load, and the calculation accuracy is relatively high, which has an irreplaceable advantage in the numerical simulation of undulating terrain and complex medium, meshfree method will provide a new reference for the numerical simulation of complex models.

- Undulating terrain seriously affects the distribution of underground electromagnetic field, it heavily changes the original anomaly characteristics. Graben topography makes TM apparent resistivity increase, and horst topography makes TM model apparent resistivity decrease. When the underground space contains abnormal body, the undulating terrain almost covers up the original abnormal information. Graben topography with a low resistivity anomaly body in different anisotropic coefficient has certain resolution, which has a reference value. In order to effectively achieve the inversion interpretation of magnetotelluric data, it becomes very important to simulate and correct the topographic effect.

\section{REFERENCES}

[1] Li Y G, Pek J, Adaptive Finite Element Modeling of Marine ControlledSource Electromagnetic Fields in Two-Dimensional General Anisotropic Media, Geophysical Journal International, 2008, 175(3), pp942- 954.

[2] Qin L J, Yang C F, Chen K, Quasi-analytic solution of 2-D magnetotelluric fields on an axially anisotropic infinite fault. Geophysical Journal International, 2013, 192(1), pp67-74.

[3] Jia X F, Hu T Y, Wang R Q, Wave equationmodeling and imaging by using element free method. Progress in Geophysics, 2006, 21(1), pp1117.

[4] Wittke J, Tezkan B, Meshfree magnetotelluric modelling, Geophysical Journal International, 2014, 198(2), pp1255-1268.

[5] Li J J, Yan J B, Developments of meshless method and applications in geophysics. Progress in Geophysics, 2014, 29(1), pp452-461. 